\title{
Experiencia y resultados en el tratamiento de la leucemia linfoblástica aguda en la edad pediátrica en el periodo comprendido entre 1989 y 2005 en la Comunidad de Navarra Experience and results of acute lymphoblastic leukaemia treatment in
children between 1989-2005 in Navarre
}

J. Molina ${ }^{1}$, T. Molins' ${ }^{1}$, F.J. Gil ${ }^{1}$, M. Sagaseta de Ilurdoz ${ }^{1}$, E. Rupérez ${ }^{1}$, E. Gembero ${ }^{1}$, F. Sala ${ }^{2}$, A. Valiente ${ }^{3}$, M.A. Labaca ${ }^{4}$

\section{RESUMEN}

Fundamento. El conocimiento de factores que se comportan como pronósticos en la leucemia linfoblástica aguda (LLA) es cada vez más importante para establecer una estrategia de tratamiento correcta. Se analiza la supervivencia global (SG), supervivencia libre de eventos (SLE) y los factores pronósticos en 16 años de experiencia en nuestra comunidad.

Material y métodos. Estudio descriptivo retrospectivo en el que se incluyen los pacientes diagnosticados de leucemia aguda (LA) en ese periodo de tiempo. Análisis uni y multivariante de aquellos factores que hemos considerado relevantes en nuestra serie aplicando el paquete estadístico SPSS para Windows versión 12.

Resultados. En el periodo comprendido entre enero de 1989 y diciembre de 2005 se diagnosticaron 58 pacientes de LA, 50 de ellos tipo linfoblástica aguda (LLA). Se analiza un subgrupo de 41 pacientes de forma más exhaustiva por ser el tipo de leucemia más frecuente y por haber estado incluidos en protocolos bien establecidos. En este grupo la SLE fue del 78\% y la SG del 87,8\%. Las variables multivariante predictoras en nuestra serie fueron: el inmunofenotipo (B-Común/Otras) con un HR de 13,82 (IC95\%: 1.019-166.008) $\mathrm{p}<0,05$, el Protocolo de la Sociedad de HematoOncología Pediátrica (SHOP) (94-99/89) con HR de 0,065 (IC95\%: $0,005-0,808) \mathrm{p}<0,02$ y la edad ( $>120$ meses/12-120meses) con un HR de 13,82 (IC95\%: 0,58-329,48) p =0,1.

Conclusiones. En nuestra serie la tasa de supervivencia es superponible a la de otros grupos colaborativos. El inmunofenotipo y el protocolo vigente se comportan como factores que influyen significativamente en la evolución de los pacientes.

Palabras clave. Leucemia linfoblástica aguda. Factores pronósticos. Protocolos tratamiento.

\begin{abstract}
Background. The determination of prognostic factors in acute lymphoblastic leukaemia (ALL) is increasingly important in establishing a correct treatment. We analyse the overall survival (OS), event free survival (EFS) and prognostic factors in our 16 years experience of treating acute lymphoblastic leukaemia.

Methods. We performed univariate and multivariate analyses of the prognostic factors we considered most significant in our serie of patients

Results. From January 1989 to December 2005, 50 cases of ALL were reported in 58 patients with LA. We analysed a subgroup of 41 patients with LLA as they were included in standard protocols. In this group the EFS was $78 \%$ and OS $87.8 \%$. Inmunophenotype is a predictor of prognosis when we compare Common with Others, with a HR of 13.82 (CI95\%: 1.019-166.008) $\mathrm{p}<0.05$; Protocol of Treatment of the Paediatric Haematology Oncology Society (SHOP) (94-99/89) with HR of 0.065 (CI95\%: $0.005-0.008) \mathrm{p}<0.02$; and Age ( $>120$ months $/ 12-120$ months) with a HR of 13.82 (CI95\%: 0.58-329.48) $\mathrm{p}=0.1$

Conclusions. The OS in our series is similar to that reported in the literature. Inmunophenotype and protocols of treatment are the most significant prognostic factors.

Key words. Acute lymphoblastic leukaemia. Prognostic factors. Treatment protocols.
\end{abstract}

1. Oncohematología Pediátrica. Servicio de Pediatría. Hospital Virgen del Camino. Pamplona.

2. Citometría. Servicio de Hematología. Hospital de Navarra. Pamplona.

3. Coitogenética Servicio de Genética. Hospital Virgen del Camino. Pamplona.

4. Servicio de Hematología. Hospital Virgen del Camino.Pamplona

Fecha de recepción el 15 de marzo de 2007

Aceptado para su publicación el 25 de mayo de 2007

Aceptación definitiva el 11 de junio de 2007

\author{
Correspondencia: \\ Correspondencia: \\ Javier Molina Garicano \\ Unidad de Oncohematología Pediátrica \\ Servicio de Pediatría \\ Hospital Virgen del Camino \\ c/ Irunlarrea, 4 \\ 31008 Pamplona \\ Tfno. 848429883 \\ Fax: 848429924 \\ E-mail: jmolinag@cfnavarra.es
}




\section{INTRODUCCIÓN}

La leucemia aguda (LA) junto con los tumores cerebrales suponen el $40-45 \%$ de la patología tumoral en la edad pediátrica. Dentro de las LA, la leucemia linfoblástica Aguda (LLA) supone casi el $80 \%$ del total. En el momento actual, entre un 70-80 \% de las LLA sobreviven a los 5 años del diagnóstico, lo que constituye uno de los mayores éxitos de la medicina ${ }^{1-4}$.

Desde el año 1989 en nuestro hospital seguimos de forma sistemática el protocolo nacional de la Sociedad de HematoOncología Pediátrica (SHOP) ${ }^{1}$, con los 3 estudios consecutivos: SHOP-89, SHOP-94 y SHOP-99. Estos estudios hacen referencia únicamente al tratamiento de las LLA, quedando excluidos por tanto los demás tipos de leucemias.

A pesar de la alta supervivencia alcanzada, en un porcentaje de pacientes la evolución es desfavorable en relación a determinados factores, que posteriormente analizaremos ${ }^{1,4,5}$.

El objetivo de este trabajo es analizar la supervivencia global de las LA durante este periodo de tiempo, concretamente de la LLA, ya que son las formas que han seguido tratamientos según protocolos bien establecidos, lo cual permite además extraer conclusiones acerca de los factores pronósticos. A pesar del escaso tamaño muestral, acorde a nuestra población infantil, los resultados que hemos obtenido son superponibles a los referidos en la literatura ${ }^{1,5}$.

\section{MATERIAL Y MÉTODOS}

Se revisaron 58 pacientes diagnosticados de LA en el Hospital Virgen del Camino de Pamplona durante el periodo comprendido entre enero de 1989 y diciembre de 2005, de los cuales 50 eran del tipo linfoblástica aguda (LLA) y 8 leucemias no linfoblásticas agudas (LNLA). De este grupo de 50 LLA, se excluyeron para el estudio detallado a 9 pacientes: 4 por debutar la enfermedad antes de los 12 meses de edad, habiendo seguido otros tratamientos, uno por haber iniciado el tratamiento según otro protocolo y otros 4 por tratarse de inmunofenotipos no incluidos en estos estudios nacionales (leucemias B maduras). Los 8 pacientes con LNLA siguieron protocolos de tratamiento nacionales de LNLA y se analizaron únicamente la SG y SLE, por tratarse de un grupo muy pequeño.

De los 41 pacientes diagnosticados de LLA se analizaron las siguientes variables: sexo, edad, número de leucocitos, hemoglobina, plaquetas, células blásticas en sangre periférica y en médula ósea (MO), cifra de lactato deshidrogenasa (LDH), visceromegalias y adenomegalias, enfermedad extramedular, inmunofenotipo, citogenética y estudio de biología molecular al diagnóstico.

El periodo de seguimiento de los casos fue muy variable, entre 14 y 192 meses, dependiendo del momento del diagnóstico.

En función de estas variables los pacientes fueron asignados a los distintos grupos de riesgo, según los protocolos vigentes en cada momento, que se resumen en riesgo estándar (RE), alto riesgo (AR) y muy alto riego (MAR) ${ }^{1}$.

Para la evaluación de resultados se tuvieron en cuenta la remisión completa (RC), la recaída, administración de radioterapia (RT) y trasplante de precursores hematopoyéticos (TPH) autólogo y alogénico.

Por último, se compararon los resultados de los tres diferentes estudios SHOP llevados a cabo a lo largo de este periodo de tiempo.

Para el análisis estadístico de los datos se utilizó el paquete informático SPSS para Windows versión 12. Se utilizaron, además del análisis descriptivo, las pruebas de análisis de supervivencia de Kaplan-Meyer, con los test estadísticos para análisis factorial (Log-rank y Breslow) así como la prueba de Regresión de Cox para análisis multifactorial.

\section{RESULTADOS}

Durante el periodo de estudio anteriormente mencionado se recogieron datos de 58 pacientes con diagnóstico de LA, siendo su edad media al diagnóstico de 69,29 meses, con una desviación estándar (DS) de 50,23 siendo la mediana de edad de 47 meses. La distribución muestra un discreto 
predominio masculino con un $58,6 \%$ de varones, sin significación estadística. En relación con la edad el 52\% de los niños eran menores de 6 años dato que se corresponde con lo descrito en la literatura ${ }^{1}$.

La procedencia de estos pacientes, según las distintas áreas geográficas de la Comunidad, se detalla en la figura 1. Teniendo en cuenta que la población Navarra de niños menores de 15 años es de 78.000 según el Departamento de Estadística de nuestra comunidad y sabiendo que la incidencia anual de LLA es de 2,7 a 3,3 pacientes por cada 100.000 niños menores de 15 años por año, el número de niños con esta patología esperados sería de 2,34 a 2,57 por año para un total de 41,12 que sumados a los correspondientes de LNLA cuya incidencia anual es de 1 por cada 100.000 niños menores de esta edad, corresponderían 12,48 en los 16 años de nuestro estudio, por tanto el total de LA esperados sería de 53 , cifra totalmente superponible a la referida por nosotros de 58 pacientes.

De los 58 pacientes, 8 presentaban LNLA siendo la mediana de seguimiento para este grupo de 16,8 meses (muestra pequeña y dispersa), la SG de $75 \%$ y la SLE de $62,5 \%$, siendo la SG media de 86 meses (IC95\%: 43-129) y la SLE de 67 meses (IC95\%: 20-113) (Fig. 2).

Los 50 pacientes restantes, afectos de LLA, fueron seguidos durante una mediana de 57,5 meses. El análisis de este grupo muestra una SG de $78 \%$ y la SLE de $70 \%$, siendo la SG media de 158 meses (IC95\%: 135-181) y la SLE de 141 meses (IC95\%: 115166) (Fig. 2).

El grupo en el que centramos nuestra atención fundamental es el de los pacientes con LLA, por ser ésta la más frecuente de leucemia en la edad pediátrica. Fueron excluidos del análisis 9 pacientes que por

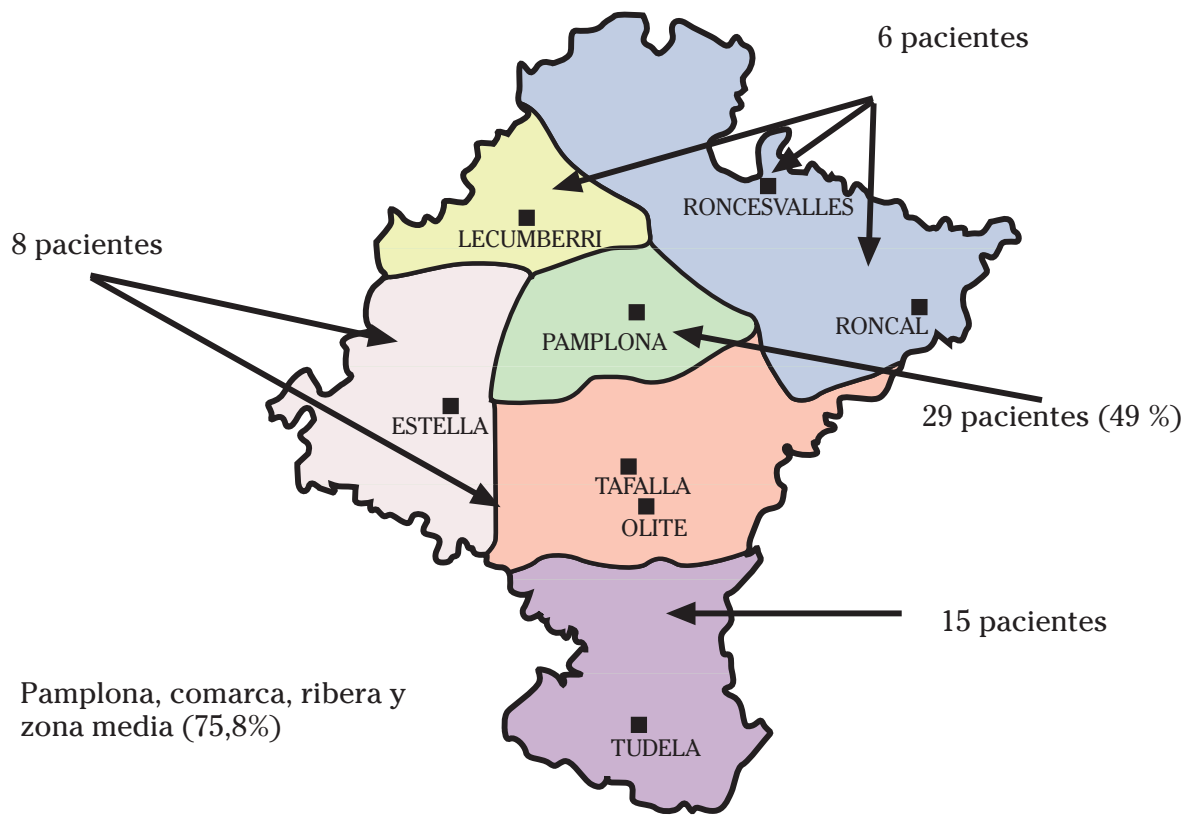

Figura 1. Distribución geográfica de la procedencia de los pacientes. 
Funciones de supervivencia

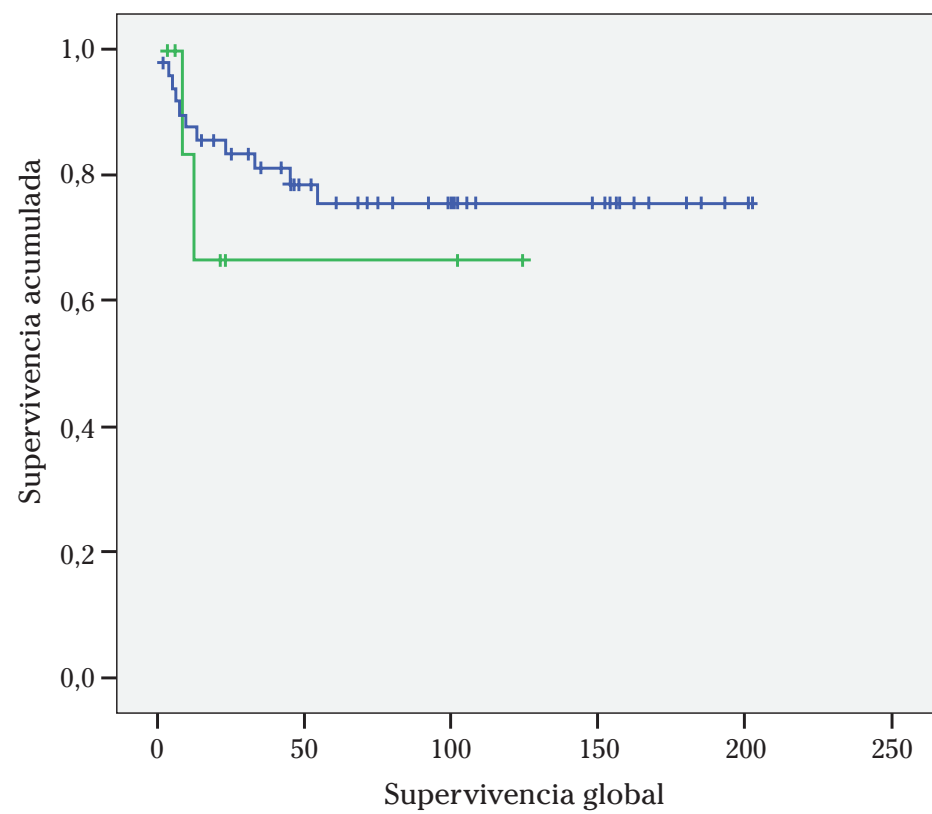

Tipo de LA

$\sqcap \mathrm{LAL}$

$\sqcap$ LANL

+ LAL-censurado

+ LANL-censurado

Figura 2. Comparativa de supervivencia de LA según estirpe celular linfoide $(\mathrm{N}=50)$ o no linfoide $(\mathrm{N}=8)$.

Tabla 1. Análisis descriptivo de las características biológicas y analíticas de los 41 pacientes con LLA en el momento del diagnóstico.

\begin{tabular}{ll}
\hline № casos $^{\circ}$ & 41 \\
Sexo & $63.4 \%$ varones $(\mathrm{V} / \mathrm{H}: 1,7 / 1)$ \\
Edad (meses) & $65,63(40,15)^{*}(23-186)^{* *}$ \\
LDH (mg/dL) & $1190.05(1604,67)^{*}(36-9850)^{* *}$ \\
Leucocitos & $80.5 \%<20.000 / \mathrm{ml} l^{* *}$ \\
Hemoglobina (g/dL) & $7,89(2,21)^{*}(4,1-13,2)^{* *}$ \\
Plaquetas (número/microlitro) & $86231,71(72190,03)^{*}(7000-262000)^{* *}$ \\
Blastos en sangre periférica (número/microlitro) & $6585,45(18396,41)^{*}(0-90000)^{* *}$ \\
Blastos en M.O (\%) & $73,85(16,65)^{*}(34-99)^{* *}$ \\
\hline
\end{tabular}

*Media (d.s) **Rango (mínimo-máximo) ***Se establece la cifra de 20.000 leucocitos como límite, según los parámetros del SHOP 99.

diversos motivos recibieron tratamientos distintos a los establecidos en los distintos protocolos de SHOP, por lo cual la población final del estudio fue de 41 pacientes, cuyas características al diagnóstico se describen en las tablas 1 y 2 .
De los 41 pacientes con LLA, 9 (22\%) presentaron eventos (no remisión o recaída): 5 fallecieron, uno de los cuales nunca alcanzó la remisión completa y cuatro tras recaídas de la enfermedad. Los otros 4 pacientes restantes fueron rescatados tras 
Tabla 2. Resumen de los factores pronósticos valorados en los 41 pacientes con LLA en el momento del diagnóstico.

\begin{tabular}{lll}
\hline № de casos & 41 & 41 \\
\hline Inmunofenotipo & LLA- B Común & $33(80,5)^{*}$ \\
& LLA- B PreB & $2(4,9)^{*}$ \\
Alteraciones citogenéticas numéricas & LLA-T & $6(14,6)^{*}$ \\
& Euploide & $18(43,9)^{*}$ \\
& Hipodiploide & $3(7,3)^{*}$ \\
Alteraciones citogenéticas estructurales & Hiperdiploide & $16(39)^{*}$ \\
& No realizada & $4(9,8)^{*}$ \\
& $\mathrm{t}(4 ; 11)$ & $1(2,4)^{*}$ \\
& $\mathrm{t}(9 ; 22)$ & $1(2,4)^{*}$ \\
Megalias y adenopatías & $\mathrm{t}(12 ; 21)$ & $2(4,9)^{*}$ \\
& $\mathrm{t}(8 ; 14)$ & $1(2,4)^{*}$ \\
Infiltración extralinfática & Otras alteraciones & $9(22)^{*}$ \\
Testículo & Ninguna & $22(52,7)^{*}$ \\
No & No realizada & $5(12,2)^{*}$ \\
\hline
\end{tabular}

${ }^{*}$ Frecuencia (\%)

Tabla 3. Variables analizadas como posibles factores pronóstico (Análisis univariante de Regresión de Cox).

\begin{tabular}{llcccc}
\hline Variable & Categorías* & LogHR & E.E & p (Sig.) & H.R \\
\hline Sexo & mujer/varon & $-0,952$ & 1,118 & 0,395 & 0,386 \\
Edad & $>120 \mathrm{~m} /<120 \mathrm{~m}$ & 1,060 & 1,121 & 0,344 & 2,887 \\
Leucocitos & $>20.000 /<20.000$ & 1,071 & 0,916 & 0,242 & 2,918 \\
LDH & $>500 /<500$ & $-0,292$ & 1,155 & 0,800 & 0,747 \\
Alteraciones citogenéticas numéricas & SÍ/NO & 0,267 & 0,913 & 0,770 & 1,306 \\
Alteraciones citogenéticas estructurales & Ś/NO & 1,107 & 0,942 & 0,240 & 3,026 \\
Inmunofenotipo & Resto/B-Comun & 2,208 & 0,928 & $\mathbf{0 , 0 1 7}$ & 9,099 \\
Megalias & SÍ/NO & 1,374 & 0,914 & 0,133 & 3,952 \\
Infiltración & SÍ/NO & 1,260 & 1,123 & 0,262 & 3,527 \\
TPH** & SÍ/NO & 0,681 & 0,913 & 0,456 & 1,975 \\
Radioterapia & SÍ/NO & 1,990 & 0,918 & $\mathbf{0 , 0 3 0}$ & 7,312 \\
Protocolos & (94+99)/89 & $-1,054$ & 0,915 & 0,250 & 0,349 \\
Recaída & Sí/NO & 2,801 & 1,118 & $\mathbf{0 , 0 1 2}$ & 16,467 \\
\hline
\end{tabular}

*En último lugar se indica la categoría de referencia

** Trasplante de progenitores hematopoyéticos

segunda remisión completa y TPH alogénico (familiar en 2 casos y no emparentado en otros 2). Todos han sobrepasado los 3 años de vida tras el evento.

En este subgrupo de 41 pacientes la SG fue de $87,8 \%$ (5 exitus) y la SLE de $78 \%$, siendo la SG media de 177 meses (IC95\%: 157-198) y la SLE de 156 meses (IC95\%: 130-
182), con una mediana de seguimiento de 71 meses (Fig. 3).

Tal y como se observa en la tabla 3 , los factores que muestran una influencia estadísticamente significativa en la supervivencia son: inmunofenotipo al diagnóstico (distinto del B-Común), precisar radioterapia y presentar una recaída de la enfermedad. 
Funciones de supervivencia

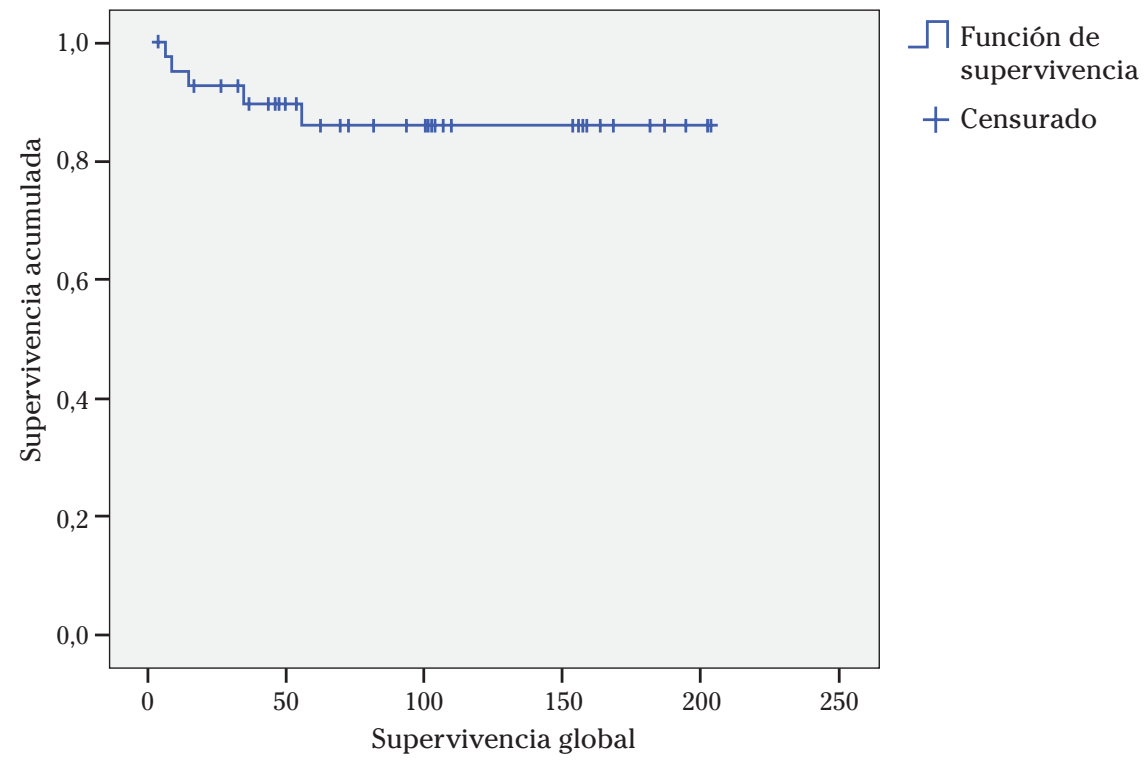

Figura 3. Curva de supervivencia global acumulada en el grupo de LLA (N=41).

Funciones de supervivencia

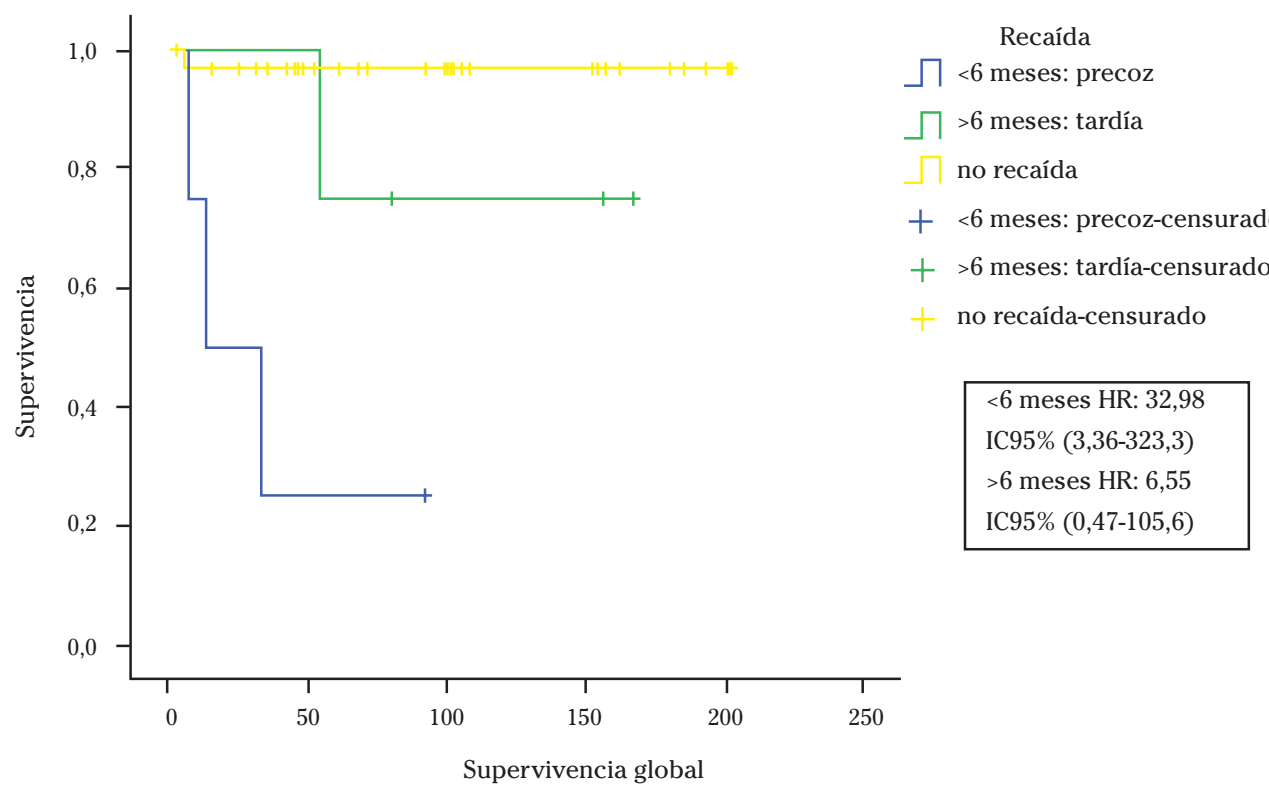

Figura 4. Función de supervivencia en LLA según el momento de la recaída N=41). 
Tabla 4. Valores pronósticos de supervivencia de las variables mediante análisis multivariante por Regresión de Cox.

\begin{tabular}{lllllr}
\hline Variable & Categorías* $^{*}$ & LogHR & E.E & p (Sig.) & \multicolumn{1}{c}{ H.R } \\
\hline Protocolos & $(94+99) / 89$ & $-2,730$ & 1,284 & $<0,034$ & 0,065 \\
Inmunofenotipo & Resto/B-Común & 2,566 & 1,299 & $<0,048$ & 13,008 \\
Edad & $>120 \mathrm{~m} / 12-120 \mathrm{~m}$ & 2,626 & 1,618 & $<0,105$ & 13,820 \\
\hline
\end{tabular}

*En último lugar se indica la categoría de referencia.

Funciones de supervivencia

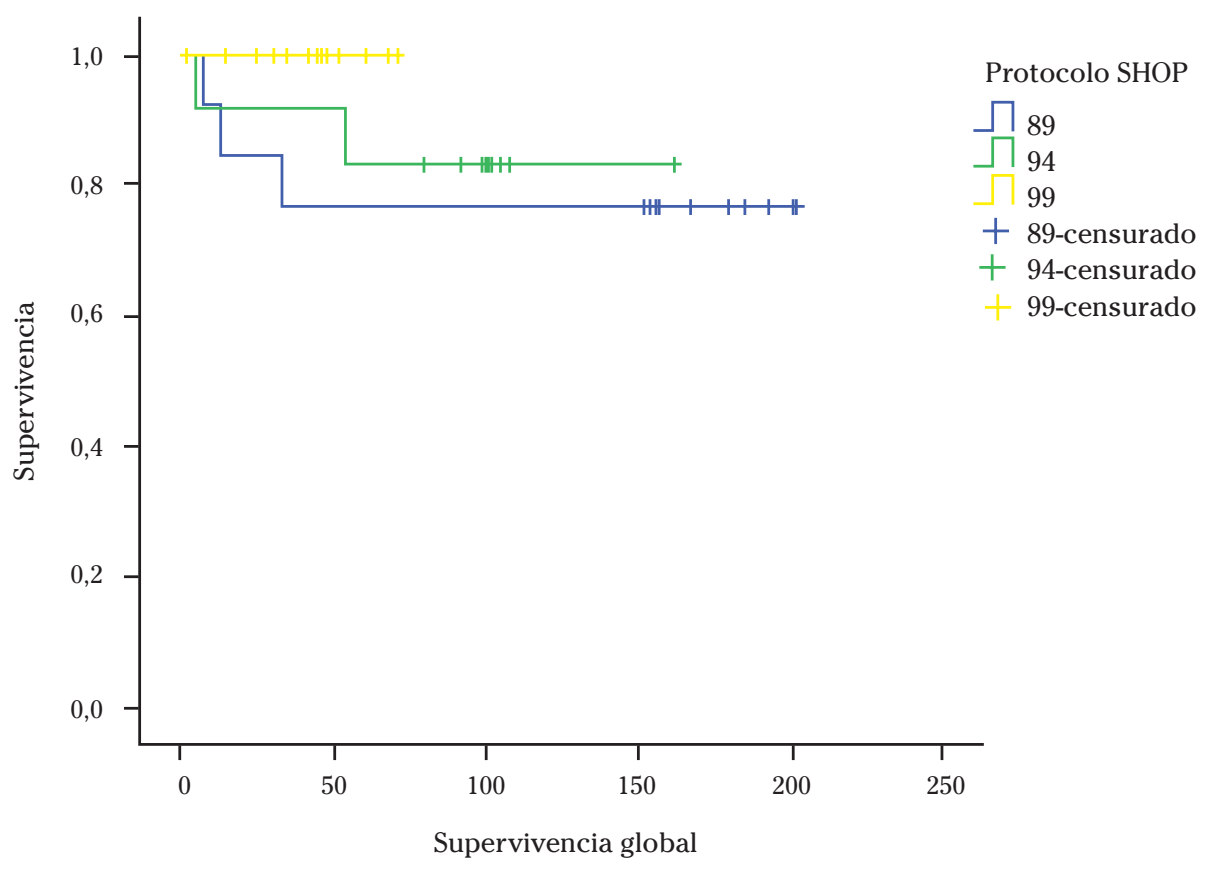

Figura 5. Supervivencia acumulada en LLA según protocolos $\mathrm{SHOP}(\mathrm{N}=41)$.

Mención aparte merecen tanto este último factor como el protocolo SHOP aplicado en los diferentes casos. Así, si dividimos la muestra en tres grupos (recaída precoz, recaída tardía y no recaída), vemos que existen diferencias muy importantes en cuanto a supervivencia según el momento de la recaída; según si se produce ésta antes o después de los 6 meses del fin del tratamiento (Fig. 4).

Se obtienen resultados similares al analizar la supervivencia según el protocolo de la SHOP aplicado (89, 94 y 99). Vemos que las curvas muestran claras diferencias en la supervivencia en los diferentes grupos, aunque éstas no sean estadísticamente significativas (Fig. 5), a favor de los que siguieron el protocolo SHOP-99.

$\mathrm{Al}$ realizar el análisis multivariante introduciendo todos los factores de riesgo pronóstico que anteriormente mencionamos, obtenemos mediante regresión de Cox un modelo que únicamente incluye dos variables significativas: protocolo (a favor del 9499) e inmunofenotipo (a favor del B común) (Tabla 4). La edad al diagnóstico se muestra próxima a la significación estadística $(\mathrm{p}=0,1)$ a favor de el rango 12-120 meses. 


\section{DISCUSIÓN}

En este trabajo hemos querido mostrar los resultados de nuestra experiencia a lo largo de los últimos 16 años en el tratamiento de la leucemia linfoblástica aguda, de acuerdo a los protocolos de la SHOP, cuyos resultados globales se han publicado recientemente ${ }^{1}$.

En nuestra serie de 41 pacientes con LLA la SG fue del $87,8 \%$ y la SLE de $78 \%$, resultados superponibles a los de otros grupos referidos en la literatura ${ }^{5-7}$. Hemos encontrado diferencias estadísticamente significativas en algunos factores estudiados mediante análisis univariante y también multivariante.

Como hemos comentado en los resultados, al realizar un análisis univariante obtuvimos resultados estadísticamente significativos al comparar según inmunofenotipo, según la necesidad de radioterapia o según la existencia o no de recaída.

Al realizar el análisis multivariante se obtuvieron solamente como significativos el inmunofenotipo y el protocolo de tratamiento utilizado, comparando el protocolo SHOP del 89 con los de 94 y 99 . El motivo de esta aparente discordancia tiene que ver muy probablemente con que las variables recogidas en gran parte del estudio están relacionadas entre sí. Al diagnóstico, en todos los pacientes se hizo una selección según riesgo, por la cual pasaban a recibir mayor o menor intensificación de tratamiento según los protocolos vigentes ${ }^{1}$. De este modo, aquellos pacientes con más factores pronósticos desfavorables al diagnóstico, recibieron un tratamiento más agresivo incluyendo en algunos casos RT profiláctica o TPH autólogo o alogénico, no siendo posible pues la aleatorización de la muestra. Por esto los resultados obtenidos en análisis univariante, tanto en significación estadística como en magnitud de HR, pueden estar sometidos a confusión. Esto hace necesario el análisis multivariante, dando quizá una cifra más realista de la importancia real de cada variable independientemente de las demás.

Los resultados que mostramos son los obtenidos en esta serie limitada por lo anteriormente referido y con datos en ocasiones no acordes a los estudios más amplios de la literatura. Prueba de ello es, por ejemplo, el que la cifra elevada de LDH tenga un valor protector estableciendo el límite en 500, dado que gran parte de los pacientes que evolucionaron favorablemente presentaban una cifra elevada al ingreso, y circunstancialmente 2 de los pacientes que fallecieron presentaban un cifra baja, sin que se pueda o se deba establecer ninguna conclusión al respecto. Otro de los factores más considerado es la cifra de leucocitos superior a 100.000 o $200.000 / \mathrm{mm}^{3}$, comportándose como variable independiente predictora de recaída, que nosotros no hemos sido capaces de constatar significativamente $\mathrm{e}^{1,5,7,8}$.

Estos resultados deben ser interpretados con cautela, dado el escaso tamaño de la muestra. De hecho, son numerosas las referencias bibliográficas que adjudican una importancia relevante como factor pronóstico en LLA a casi todas las variables introducidas en este análisis y que en nuestro caso no son incluidas en el modelo final, muy probablemente por un problema de potencia estadística.

En relación a la enfermedad residual hay diversas publicaciones que sin lugar a duda establecen un valor predictivo de recaída de la enfermedad y cuya determinación se incluye actualmente en el protocolo. Junto con la detección de reacción en cadena de la polimerasa a tiempo real serán una prometedora estretegia ${ }^{4,5,9,10}$.

Al realizar el análisis de la influencia de los factores de riesgo y factores pronósticos de la enfermedad, debemos contemplar el problema del tamaño muestral en los estudios oncológicos. La baja incidencia de este tipo de enfermedades unida a la pequeña población infantil, hace que la sensibilidad estadística de las pruebas sea muy reducida y por tanto muchas de las variables estudiadas no arrojan resultados estadísticamente significativos. Pese a todo, exponemos a continuación el análisis uni y multivariante de los distintos factores recogidos en nuestro estudio, con sus valores de Hazar Ratio (HR), que pueden orientarnos sobre el peso que ejercen en la supervivencia las diferentes variables recogidas antes, durante y después del tratamiento (Tabla 3). 
El TPH alogénico como estrategia terapéutica en caso de recaída es considerada como la idónea por otros autores y deja prácticamente olvidado el trasplante antólogo, dado el riesgo conocido de poder inocular de nuevo la alteración genética que provocó la leucemia inicial $1^{1,5,11-13}$.

Como conclusión pensamos que aun siendo nuestra serie pequeña, el formar parte de protocolos cooperativos nacionales nos hace seguir terapias muy actuales y confirmar la supervivencia al compararla con los registros nacionales. Esto indica claramente que se debe trabajar dentro de grupos cooperativos rigurosamente supervisados, para conseguir cada vez mejores resultados y menos efectos secundarios.

Es evidente que falta por determinar de manera estricta factores pronósticos predictivos de recaída y adecuarlos a los grupos de riesgo que se establezcan en los protocolos de tratamiento, con el fin de lograr un mayor porcentaje de curación en la leucemia linfoblástica aguda en la edad pediátrica.

Recientemente se ha introducido el concepto de enfermedad mínima residual como uno de los factores más importantes para la respuesta al tratamiento, pero este factor no ha sido analizado en nuestra serie, puesto que sólo se ha determinado en los pacientes que se han tratado según el último protocolo nacional.

\section{BIBLIOGRAFÍA}

1. Badell I, Cubells J. Resultados terapéuticos en la leucemia linfoblástica aguda infantil con los protocolos SHOP de la Sociedad Española de Hematología Pediátrica (SEHP) y la Sociedad Española de Oncología Pediátrica (SEOP). 1989-2005. Rev Esp Pediatr 2005; 61: 273-281.

2. Puy CH, Relling MV, Downing JR. Acute lymphoblastic leukaemia. N Engl J Med 2004; 350: 1535-1548.

3. Cubells J, Badell I. Tratamiento de la leucemia linfoblástica aguda. En: Fernández Delgado R, Molina Garicano J. Eds. Leucemia linfoblástica aguda en la infancia. Jarpyo Editores. Madrid 1995; 97-105.

4. Vergara B, Svarch E et al. Leucemia linfoblástica aguda infantil. Seguimiento postfinalización del tratamiento en 430 pacientes. Rev Esp Pediatr 2004; 60: 348-354.
5. Pérez A, Alonso M, Ramírez J, Contra T, MadeRo L, DíAz MA. Veinte años de experiencia en el tratamiento de la leucemia linfoblástica aguda. An Pediatr 2006; 65: 198-204.

6. Reiter A, Schrappe M, LudWing WD, Hiddeman W, Sauter S, Henze G et al. Chemotherapy in 998 unselected childhood acute lymphoblastic leukaemia patients. Results an conclusions of the multicenter trial ALL-BFM 86. Blood 1994; 84: 3122-3133.

7. Schrappe M, Reiter A, SAuter S, LudWing WD, Worman B, HaRbott J et al. Concept an interin result of the ALL-BFM 90 therapy study in treatment of acute lymphoblastic leukaemia in children and adolescents: The significance of initial therapy response in blood and bone marrow. Klin Padiatr 1994; 206: 208-221.

8. Schrappe M, Reiter A, Zimmermann M, HarbotT J, Ludwing WD, HENZE G et al. Long term results of four consecutive trials in childhood ALL performed by the ALL-BFM study group from 1981 to 1995. Berlin-FrankfurtMünster. Leukaemia 2000; 14: 2205-2222.

9. Dworzak Mn, Fröschl G, Printz D, Mann G, Pötschger U, MüHLEgGer N et al. Prognostic significance and modalities of flow cytometric minimal residual disease detection in childhood acute lymphoblastic leukaemia. Blood 2002; 99: 1952-1958.

10. Bastida P, Palacio C, Solsona L, Ortega J, SÁNCHEZ dE TOlEDo J. Minimal residual disease in acute lymphoblastic leukaemia: a new concept of complete remission. An Pediatr 2005; 63: 390-395.

11. Matsuzaki A, Nagatoshi Y, Inada H, Nakayama $\mathrm{H}$, YANAi F, AYUKaWA $\mathrm{H}$ et al. Prognostic factors for relapsed childhood acute lymphoblasctic leukaemia: impact of allogeneic stem cell transplantation- a report from the KyushuYamaguchi Children's Cancer Study Group. Pediatr Blood Cancer 2005; 45: 111-120.

12. Bunin N, Aplenc R, Kamani N, Shaw K, CnaAn A, Simms S. Randomized trial of busulfan vs Total body irradiation contain conditioning regimens for children with acute lymphoblastic leukaemia: A Pediatric Blood and Marrow Transplant Consortium study. Bone Marrow Transplant 2003; 32: 543-548.

13. Bunin N, Carston M, Wall D, Adams R, Casper J, Kamani N, King R. National Marrow Donor Program Working Group. Unrelated marrow transplantation for children with acute lymphoblastic leukaemia in second remission. Blood 2002; 99: 3151-3157. 
